\title{
Fluorescence Polarization for Monitoring Ribozyme Reactions in Real Time
}

BioTechniques 29:344-351 (August 2000)

\author{
K.K. Singh, T. Rücker, A. \\ Hanne, R. Parwaresch and \\ G. Krupp \\ Institute for Hematopathology, \\ University of Kiel, Kiel, \\ Germany
}

\begin{abstract}
Fluorescence polarization has been used recently to monitor diverse macromolecular interactions. In this report, the application of fluorescence polarization has been extended to monitor ribozyme reactions in real time. With fluorescently labeled substrate RNAs, group I ribozyme ligation and hammerhead ribozyme cleavage reactions were studied by fluorescence polarization in substrate excess (multiple turnover) conditions. These results also show that fluorescently labeled RNAs remain active substrates for ribozymes. Furthermore, a direct comparison of fluorescence polarization with fluorescence resonance energy transfer showed that both techniques were comparable for monitoring ribozyme reactions.
\end{abstract}

\section{INTRODUCTION}

Ribozymes are oligoribonucleotide sequences that catalyze various reaction types with multiple turnover (like protein enzymes). They occur in both cis (intramolecular) and trans (intermolecular) configurations. Ribozymes are considered potential antisense tools to inhibit the expression of pathogenic sequences or mutated genes (e.g., viral pathogens such as HIV, hepatitis virus and cancer-causing oncogenes) $(5,14$, 15,21). However, these applications are hampered by the fact that conventional methods for studying ribozyme kinetics are cumbersome, time consuming and often include radioisotope labeling of RNAs. Characterization of ribozyme reactions using fluorescence detection techniques can be used conveniently for this purpose. Also, nonradioactive reagents do not create health hazards and problems of waste disposal. With recently improved fluorescence instrumentation, all measurements can be achieved in one tube with no need for direct handling of the samples (20). Furthermore, if needed, the reaction end products can be analyzed by a fluorimager after polyacrylamide gel electrophoresis.

Fluorescence resonance energy transfer (FRET) has already been used for studying diverse macromolecular interactions including ribozyme cleavage kinetics in real time (20). Fluorescence polarization is another fluorescence-based technique $(12,18)$; it is considered an excellent tool for studying cellular and molecular biology of macromolecules $(1,3)$, such as nucleic acid analysis in homogeneous conditions $(4,7,17,22-24)$, nucleic acid cleavage by RNase (2) and DNase (26), protein-protein and protein-DNA interactions (11), homogeneous genotyping (10) and also for drug-level monitoring using immunoassays (13).

Fluorescence polarization can be observed when fluorescent molecules are excited with plane-polarized light (19, 25 ). The direction in which a molecule absorbs light is the excitation dipole, and the direction in which the molecule emits light is the emission dipole. If the direction of all dipoles is parallel to the plane of the exciting plane-polarized light (and the molecules do not rotate), the observed polarization would be $1000 \mathrm{mP}$. If the excitation dipoles are arranged randomly, the molecules absorb and emit light to varying degrees, and the resulting polarization, called limiting polarization $\left(\mathrm{P}_{0}\right)$, will be 500 $\mathrm{mP}$. In practical applications, the molecules will be in solution and thus rotate and tumble during the period between fluorophore excitation and light emission. Therefore, the plane of emitted light will be different from the plane of excitation. The light becomes partially depolarized such that the emitted light has both vertical $\left(\mathrm{V}_{\mathrm{i}}\right)$ and horizontal components $\left(\mathrm{H}_{\mathrm{i}}\right)$, that is, fluorescence polarization $=\left(\mathrm{V}_{\mathrm{i}}-\mathrm{H}_{\mathrm{i}}\right) /\left(\mathrm{V}_{\mathrm{i}}+\mathrm{H}_{\mathrm{i}}\right)$.

Since it is impossible to get a polarization value greater than the limiting polarization, values higher than $500 \mathrm{mP}$ indicate either the presence of scattered light or incorrect calibration of the system. Therefore, polarization values should be between 0 and $500 \mathrm{mP}$ for all experimental observations. If temperature and viscosity are constant, fluorescence polarization is directly dependent only on the molecular volume, and it 
can be used to study any kind of association or dissociation (degradation) of molecules $(12,18)$.

Anisotropy is often used instead of fluorescence polarization. Both polarization (P) and anisotropy (A) values are derived from the measured vertical and horizontal intensities and can be easily interconverted with the equation $\mathrm{A}=2 \mathrm{P} /(3-\mathrm{P})$. Anisotropy values are preferred because they are additive and more convenient for mathematical conversions. Throughout this text, we have used anisotropy values, whereas we retain fluorescence polarization to describe this technique.

\section{MATERIALS AND METHODS}

\section{Design and Synthesis of Ribozymes and Substrates}

For monitoring ribozyme reactions by fluorescence polarization with single fluorescently labeled RNAs, the sunY ribozyme (a shortened derivative of sunY self-splicing group I intron) was used. It catalyzes a phosphodiester exchange reaction that leads to RNA ligation with concommitant release of guanosine (8). This ribozyme was obtained by in vitro transcription from a plasmid (8). The corresponding two- and three-subunit fluorescent substrate RNAs $(8,9)$ were chemically synthesized (NAPS Göttingen $\mathrm{GmbH}$, Göttingen, Germany). Different ligands were also used for characterization of fluorescence polarization for ribozyme reactions.

To monitor hammerhead ribozyme cleavage reactions with double fluorescently labeled RNAs, a modified version of the hammerhead ribozyme-substrate system recently described by Clouet-D'Orval and Uhlenbeck (6) was used (Figure 2A). The RNA substrate was 3'-labeled with FAM (6-carboxyfluorescein, absorption at $488 \mathrm{~nm}$ and emission at $515 \mathrm{~nm}$ ) next to a uridine via FAM-CPG during solid phase chemical synthesis. The 5'-end-labeling with TAMRA (tetramethylrhodamine, absorption at $555 \mathrm{~nm}$ and emission at $580 \mathrm{~nm}$ ) was done with an extra thymidine deoxynucleotide (dT) through an amino linker at C-5 of the base moiety. All oligoribonucleotides were purchased from NAPS Göttingen $\mathrm{GmbH}$.

\section{Monitoring Ribozyme Reactions by Fluorescence Polarization Using Single Fluorescently Labeled RNAs}

To monitor cleavage and ligation reactions by sunY ribozyme, a two-part substrate with a single fluorescently labeled RNA was used (Figure 1A, upper panel) such that the large RNA (P1) was always 5'-FAM labeled, and different ligands (biotin/streptavidin-biotin) were attached to the small RNA (P1P) at position L. As another option in this series, a second fluorescent dye (TAMRA) was present at position L. Substrates $(4 \mu \mathrm{M}$; $\mathrm{P} 1 \mathrm{P}$ and $\mathrm{P} 1)$ and $1.5 \mu \mathrm{M}$ ribozyme were added to $30 \mathrm{mM}$ Tris- $\mathrm{HCl}$ (pH 7.4), 400 $\mathrm{mM} \mathrm{KCl}, 10 \mathrm{mM}$ ammonium chloride and $10 \%$ ethanol. A $50-\mu \mathrm{L}$ reaction was started at $25^{\circ} \mathrm{C}$ by adding $150 \mathrm{mM}$ $\mathrm{MgCl}_{2}$, and readings were taken every 3 min in fluorescence mode by POLARstar (BMG LabTechnologies, Offenburg, Germany).

\section{Monitoring Ribozyme Reactions Simultaneously by Fluorescence Polarization and FRET Using Double Fluorescently Labeled RNAs}

To monitor cleavage reactions, a $50-\mu \mathrm{L}$ hammerhead ribozyme reaction contained 5 pmol substrate and decreasing concentrations of ribozyme $(0.3,0.1,0.03 \mathrm{pmol})$ in $50 \mathrm{mM}$ Tris$\mathrm{HCl}(\mathrm{pH} 7.5)$ and $10 \%$ ethanol. The reaction mixture was transferred to a 96-well plate (Corning Costar, Corning, NY, USA) and was preincubated for $15 \mathrm{~min}$ at $37^{\circ} \mathrm{C}$. The reaction was started by adding $20 \mathrm{mM} \mathrm{MgCl}$, and measurements were taken every $3 \mathrm{~min}$ for 100 cycles. Time-dependent changes in fluorescence intensity and fluorescence polarization were recorded simultaneously in fluorescence polarization mode by POLARstar.

To monitor a group I ribozyme-catalyzed ligation reaction, a three-part substrate with one double fluorescently labeled RNA (consisting of 5'-FAMUGATCTGT-TAMRA-3', CCCUCU and GGGAGGUAGACA, as shown in Figure 3A) (9) was used. For this purpose, the substrate was changed from the originally reported substrate (9) so that, for attaching FAM, an extra U was added next to G; otherwise, FAM fluorescence would be quenched signifi- 
cantly by the adjacent G-base (20). Also, several $U$ positions were replaced by dT and at the $3^{\prime}$-end, an extra dT was inserted between $\mathrm{G}$ and the TAMRA label; again, this separation of dye and G-base was required (20). The $50-\mu \mathrm{L}$ ligation reactions were performed as previously described (8). Briefly, $5 \mu \mathrm{M}$ each of three-part substrate and $1 \mu \mathrm{M}$ sunY ribozyme were incubated in $30 \mathrm{mM}$ Tris- $\mathrm{HCl}$ ( $\mathrm{pH}$ 7.4), $400 \mathrm{mM} \mathrm{KCl}, 10 \mathrm{mM}$ ammonium chloride and $10 \%$ ethanol. The reaction was started by adding $150 \mathrm{mM} \mathrm{MgCl} 2$ at $25^{\circ} \mathrm{C}$ and read every $5 \mathrm{~min}$ for $140 \mathrm{~min}$ by POLARstar as described above.

\section{RESULTS AND DISCUSSION}

POLARstar is a multi-well plate reader that monitors changes in both fluorescence intensity and fluorescence polarization. Reagent injection and incubation steps are fully programmable, and the maximum temperature is about $40^{\circ} \mathrm{C}$ because it is an open plate reader. It can read within a wavelength range of $250-900 \mathrm{~nm}$ for standard fluores- cence and 350-900 nm for fluorescence polarization with a high sensitivity of about 5-10 nM fluorescently labeled substrate.

\section{Monitoring Ribozyme Reactions by Fluorescence Polarization Using Single Fluorescently Labeled RNAs}

All polarization readings were obtained in $\mathrm{mP}$ units during direct measurements and were changed to anisotropy units, which are easier to manipulate mathematically. Single fluorescently labeled substrate RNAs were used for studying cleavage reactions by the sunY ribozyme, which cleaves the large substrate RNA and concomitantly ligates both RNA substrates (Figure 1A). We designed the fluorescent substrate RNAs for the sunY ribozyme in two parts so that one fluorescent RNA (P1, the larger RNA) always carried the FAM fluorophore at the 5'-end, and the other RNA (P1P) could be labeled with various ligands at its 5 '-end (Figure 1A). In the presence of the sunY ribozyme, the FAM-UUG moiety is cleaved from the FAM-labeled RNA
(P1), leading to a decrease in anisotropy caused by a decrease in the molecular volume of the FAM-labeled oligonucleotide. The remainder of the P1 cleavage product is ligated to the second substrate RNA (P1P). Since we monitored only FAM fluorescence, we only observed the decrease in anisotropy of the FAM-labeled P1 substrate RNA caused by the release of the small FAM-UUG.

Results in curves a and b (Figure 1B) show the anisotropy signal in the absence and presence of ribozyme, respectively, obtained with the FAM-labeled large RNA subunit (FAM-P1) and unlabeled P1P RNA. In this cleavage reaction with a single fluorescently labeled RNA by the sunY ribozyme, we show that with the release of FAMUUG, anisotropy decreased concurrently with the decrease in molecular volume. After the attachment of biotin or biotin-streptavidin to the 5 '-end (position L) of P1P (curves e and g, respectively), a decrease in anisotropy values was observed in the presence of ribozyme (curves $f$ and $h$, respectively). Moreover, when TAMRA was attached to the small RNA subunit (TAMRA-

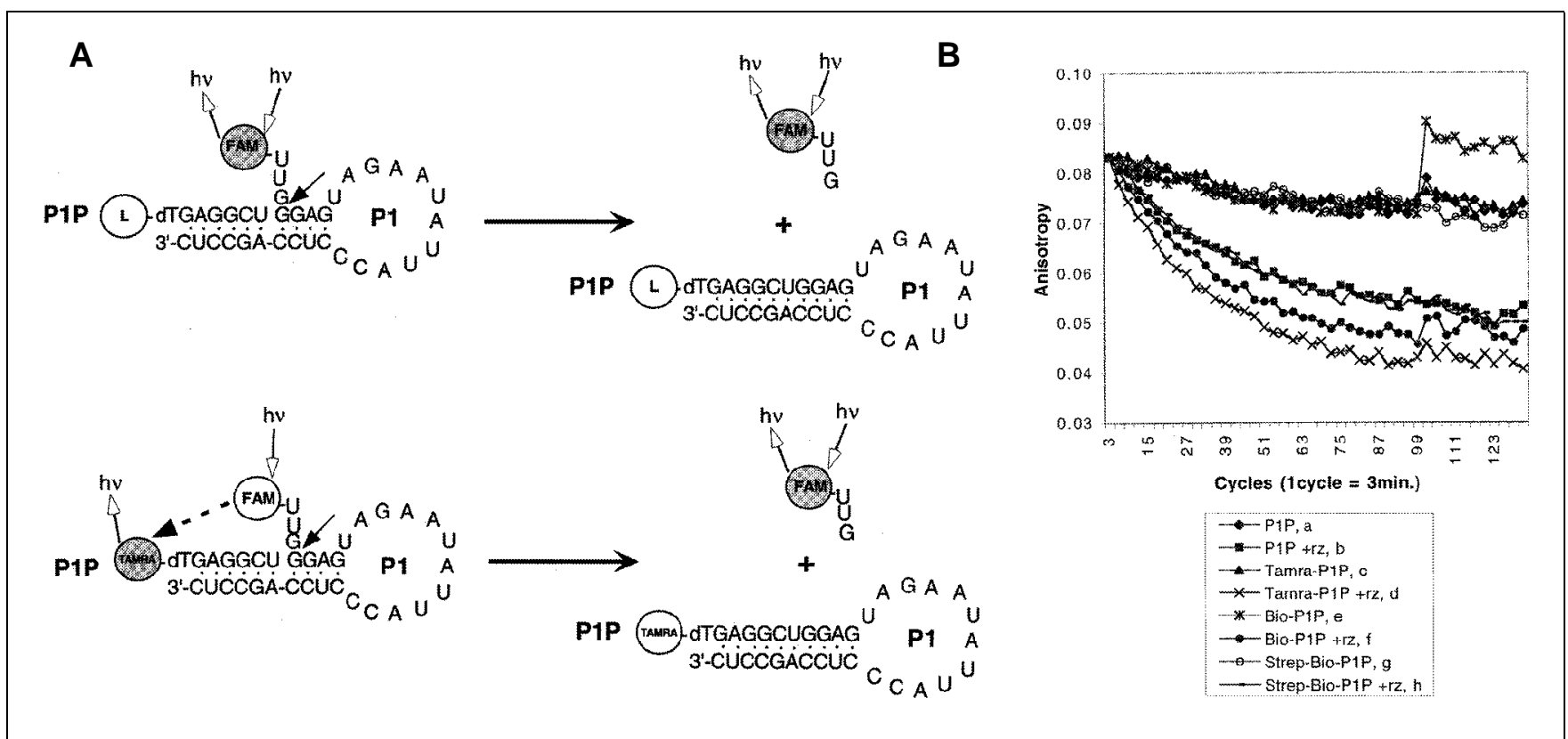

Figure 1. Monitoring sunY ribozyme reactions using single fluorescently labeled RNA. (A) Sequences and structure of two-part RNA substrates (P1 and P1P) are shown. Sites for the attachment of the fluorescent dye (FAM) and other ligands (L) are marked with circles. Unfilled arrows indicate the absorption and emission of light from the fluorophore. The solid, filled arrow indicates the site of cleavage by the sunY ribozyme. Note that in the absence of a second fluorescent dye (upper panel), light is absorbed and emitted by FAM before and after the cleavage. When TAMRA is attached at position L (lower panel), incoming light is absorbed by FAM but its fluorescence is quenched and thus emitted by TAMRA. After cleavage, quenching by TAMRA is released. However, the presence of an extra fluorescent dye did not significantly affect the anisotropy values obtained for the cleavage reactions (see text). (B) Changes in anisotropy after cleavage of singly labeled or doubly labeled (with TAMRA) fluorescent RNA substrates are shown. Note that all anisotropy values were normalized with respect to the control substrate without ribozyme (rz). 
P1P), the cleavage resulted in a slightly larger decrease in anisotropy in the presence of ribozyme as shown in curve $d$ (in agreement with the slightly increased molecular volume of TAMRA-labeled substrate).

Hence, the addition of another fluorescent dye (TAMRA) did not alter the reaction because the differences of the quenched reaction curves $\mathrm{c}$ and $\mathrm{d}$ (TAMRA-P1P) and the unquenched reaction curves e and $\mathrm{f}$, respectively (Bio$\mathrm{P} 1 \mathrm{P})$, are only minor. This showed that attachment of TAMRA, biotin or streptavidin-biotin to the RNA substrate did not interfere with the active substrate function, and specifically designed ribozymes can be used to cleave ligandbound RNAs for various applications (e.g., purification of ligand-bound RNAs). Furthermore, after 33 readings (99 min), $10 \mu \mathrm{L} 20 \mu \mathrm{M}$ streptavidin (or water for streptavidin-biotin sample) were added to all the reactions, and measurements were continued to see if the increase in the molecular volume of biotin-P1P after binding of streptavidin was reflected in an increased anisotropy value. This result was indeed observed (see curve e after 33 cycles).

Consequently, the difference in the fluorescene polarization of substrate versus products was enlarged. At the beginning of the reactions, all substrate RNA molecules were intact, and the initial anisotropy values were high. At the end of the reactions, the FAM-labeled product FAM-UUG was released and because of the small molecular size, anisotropy values were lower. Anisotropy values between these extremes represent a mixture of intact and cleaved substrates. Control substrate without ribozyme did show a small decrease in anisotropy during the incubation period (300 min). We generally observed this behavior with fluorescently labeled RNAs. This may indicate that a

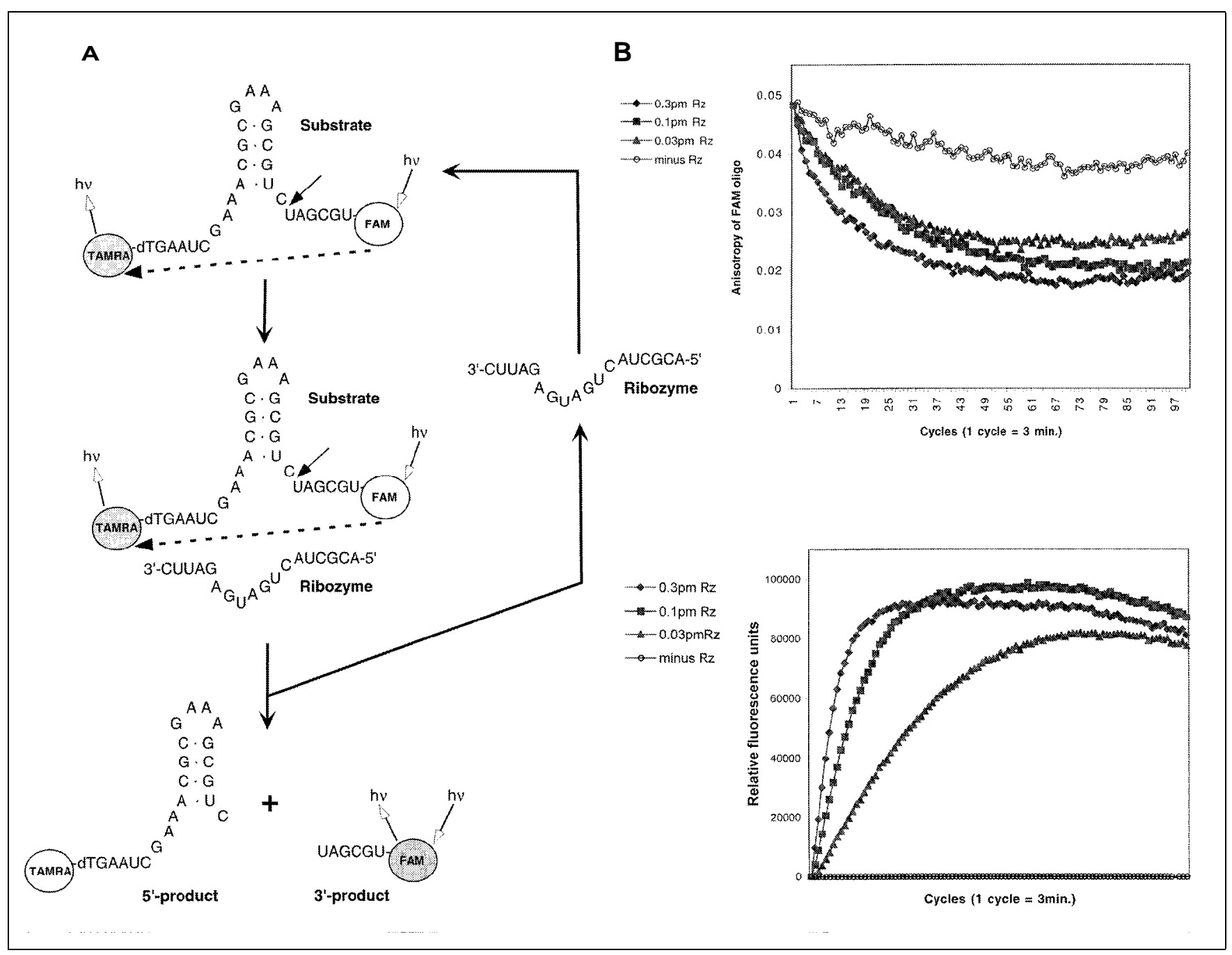

Figure 2. Monitoring hammerhead ribozyme cleavage reactions using double fluorescently labeled RNA. (A) Sequences and structure of the hammerhead ribozyme and corresponding substrate are shown. The mechanism for monitoring the cleavage reaction by FRET is indicated. FRET occurs in intact substrate, that is, fluorescence of FAM is quenched by intramolecular energy transfer to TAMRA. After cleavage of the substrate by the trans-acting ribozyme, both RNA products dissociate from the ribozyme, and fluorescence from FAM is restored. The resulting change in FAM fluorescence can be used to monitor cleavage reactions in real time. Only the cleavage products that have dissociated from the ribozyme are shown in the last step. This dissociation is fast and not rate limiting (20). (B) Changes in anisotropy (upper panel) and fluorescence intensity (lower panel) as monitored by fluorescence polarization and FRET, respectively, are shown for ribozyme (Rz)-catalyzed cleavage reactions. 
fraction of the fluorescently labeled RNA forms multimolecular aggregates that dissociate slowly, leading to a decrease in anisotropy. Substrate RNAs of different molecular weights (because of different ligands attached) show the same initial anisotropy (Figure 1B) because their values have been normalized so that they can be compared for different reactions. Final anisotropy values for all reactions in the presence of ribozyme show a reasonable variation of $10 \%-20 \%$.

\section{Monitoring Ribozyme Reactions by Fluorescence Polarization Using Double Fluorescently Labeled RNAs}

Since the attachment of two fluorescent dyes to the two-part substrate RNA did not interfere with fluorescence polarization monitoring of the cleavage/ligation reaction with the sunY ribozyme, we used a double fluorescently labeled substrate RNA to study cleavage reactions with a hammerhead ribozyme. Using these substrates, simultaneous fluorescence polarization and FRET measurements could be obtained. Fluorescence polarization measurements (Figure 2B upper panel) clearly showed a decrease in anisotropy after site-specific, ribozyme-catalyzed cleavage of FAM-labeled substrate RNA to a FAM-labeled hexanucleotide. The increase in observed cleavage rate was proportional to the corresponding increase in ribozyme concentrations, reflecting a quantitation of the ribozyme reaction. These reactions with excess substrate resulted in multiple turnover cleavage by ribozyme because 5 pmol substrate were completely cleaved by $0.1 \mathrm{pmol}$ ribozyme. Hence, 50 substrate molecules were cleaved in approximately $100 \mathrm{~min}$ by one ribozyme molecule at a rate of 0.5 molecules/min.

Similar cleavage kinetics were obtained in the FRET measurements (Figure $2 \mathrm{~B}$, lower panel) in which the increase in FAM fluorescence was observed when quenching from TAMRA was relieved after cleavage of the substrate. The apparent maximum (for FRET) or minimum (for fluorescence polarization) fluorescence signals indicated the completion of the reaction. This was confirmed by polyacrylamide gel electrophoresis of these reaction products, followed by analysis with a fluorimager (Storm ${ }^{\mathrm{TM}}$ 860; Amersham Pharmacia Biotech, Piscataway, NJ, USA). Alternatively, the fluorescent substrates were $5^{\prime}$-end labeled with $\gamma$ [32P]-ATP (fluorescent labels at the $5^{\prime}$ end were attached using an amino linker at the base moiety, leaving the $5^{\prime}-\mathrm{OH}$ group free for $5^{\prime}$-end radioactive labeling), and reaction products were analyzed by both fluorimager and phosphorimager modes of Storm 860 (data not shown).

In addition, a group I intron (sunY) ribozyme-catalyzed ligation reaction was studied with a double fluorescently labeled three-part substrate RNA (Figure 3A). Simultaneous FRET and anisotropy values were obtained (Fig- ure 3B). Anisotropy values for FAM-labeled RNA decreased as FAM-UG was cleaved from the fluorescent oligonucleotide (with the decrease in the molecular volume), concomitant with the ligation of the two RNA parts of the substrate. In the case of FRET, the FAM fluorescence intensity increased during the reaction when $5^{\prime}$-FAM-UG was liberated with the consequent release of quenching by TAMRA. Here, both FRET and fluorescence polarization showed similar relative changes in their values (i.e., about 1.5 times the increase in FAM fluorescence and 1.5 times the decrease in anisotropy value) (Figure 3B). High concentrations of substrate were necessary to monitor ligation reactions of the three-part RNA substrates (9), but, with a single-unit

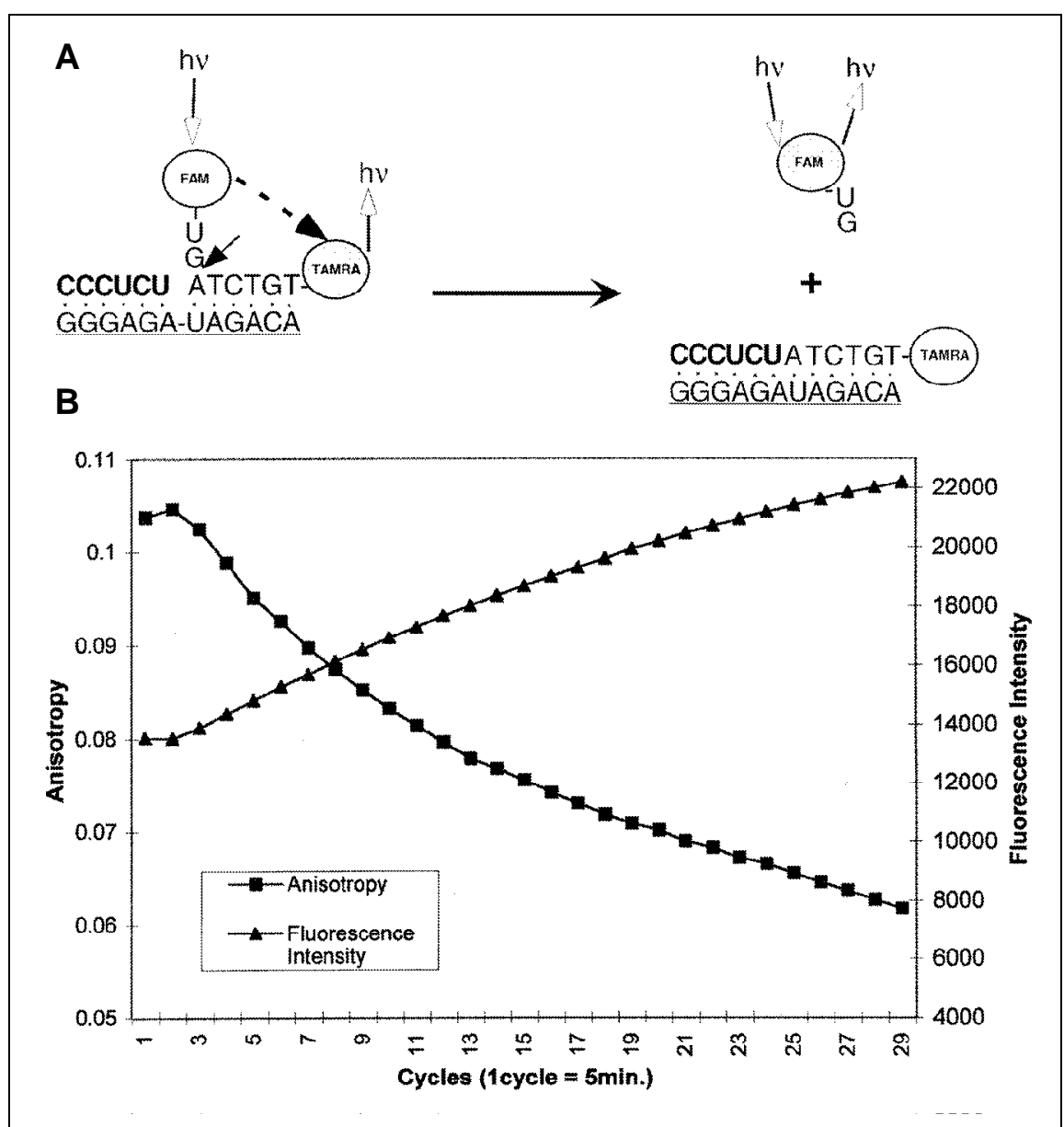

Figure 3. Monitoring sunY ribozyme reactions by simultaneous fluorescence polarization and FRET measurements using double fluorescently labeled RNA. (A) Sequences and structure of a three-part substrate with one double fluorescently labeled RNA are shown. The first substrate RNA is shown in bold letters, the second substrate RNA with the fluorescent dyes is shown as plain text and the third substrate RNA is underlined. (B) Monitoring of ribozyme ligation reaction by simultaneous measurements of fluorescence polarization (anisotropy values) and of FRET (fluorescence intensity). 
RNA substrate for the hammerhead ribozyme, as low as $5 \mathrm{nM}$ fluorescently labeled substrate could be used (data not shown). In principle, anisotropy values obtained with double fluorescently labeled substrates are affected by fluorescence quenching (16), but these effects are minor since fluorescence intensities changed less than twofold.

Moreover, we have observed that differences between anisotropy of unquenched (singly labeled) and quenched (doubly labeled) fluorescent RNAs were really minor (Figure $1 \mathrm{~B}$ ) with our substrate-ribozyme systems; therefore, quench corrections were not included. Fluorescence quench corrections will be necessary in systems with double fluorescently labeled RNAs in which initial and final fluorescence intensities show drastic changes (16). Here, we used double fluorescently labeled substrate RNAs to directly compare the results of fluorescence polarization and FRET. Normally, for fluorescence polarization studies, only single fluorescently labeled RNAs will be used when fluorescence quenching is no issue.

Fluorescence polarization is a direct, simple, fast, sensitive and nonradioactive homogeneous method that requires only single fluorescently labeled oligoribonucleotides. The power of fluorescence polarization for various applications lies in the fact that fluorescence polarization is independent of the intensity of the fluorescent signal. Hence, the assays are easy to construct because the fluorophores do not have to respond to any reaction by undergoing any change in fluorescence intensity. Also, fluorescence polarization is relatively insensitive to instrument parameters such as drift, gain settings and lamp changes. Our results demonstrated that ribozyme-catalyzed cleavage and ligation reactions can be monitored with fluorescence polarization.

\section{ACKNOWLEDGMENTS}

This work was supported by KinderKrebs-Initiative Buchholz, Germany, and Intelligene Ltd., Jeruslem, Israel. K.K.S. was supported by postdoctoral fellowship from the Alexander-vonHumboldt Foundation, Bonn, Germany.

\section{REFERENCES}

1.Belford, G.G., R.L. Belford and G. Weber. 1972. Dynamics of fluorescence polarization in macromolecules. Proc. Natl. Acad. Sci. USA 69:1392-1393.

2.Bolger, R. and D. Thompson. 1994. A quantitative RNase assay using fluorescence polarization. Am. Biotechnol. Lab. 12:113-116.

3.Checovich, W.J., R.E. Bolger and T. Burke. 1995. Fluorescence polarization-a new tool for cell and molecular biology. Nature 375:254-256.

4.Chen, X., L. Levine and P.Y. Kwok. 1999. Fluorescence polarization in homogeneous nucleic acid analysis. Genome Res. 9:492498.

5.Christoffersen, R.E. and J. Marr. 1995. Ribozymes as human therapeutic agents. J. Med. Chem. 38:2023-2037.

6.Clouet-D'Orval, B. and O.C. Uhlenbeck. 1996. Kinetic characterization of two I/II format hammerhead ribozymes. RNA 2:483-491.

7.Devlin, R., R.M. Studholme, W.B. Dandliker, E. Fahy, K. Blumeyer and S.S. Ghosh. 1993. Homogeneous detection of nucleic acids by transient-state polarized fluorescence. Clin. Chem. 39:1939-1943.

8.Doudna, J.A., S. Couture and J.W. Szostak. 1991. A multisubunit ribozyme that is a catalyst of and template for complementary strand RNA synthesis. Science 251:1605-1608.

9.Doudna, J.A., N. Usman and J.W. Szostak. 1993. Ribozyme-catalyzed primer extension by trinucleotides: a model for the RNA-catalyzed replication of RNA. Biochemistry 32:2111-2115.

10.Gibson, N.J., H.L. Gillard, D. Whitcombe, R.M. Ferrie, C.R. Newton and S. Little. 1997. A homogeneous method for genotyping with fluorescence polarization. Clin. Chem. 43:1336-1341.

11.Heyduk, T., Y. Ma, H. Tang and R.H. Ebright. 1996. Fluorescence anisotropy: rapid, quantitative assay for protein-DNA and protein-protein interaction. Methods Enzymol. 274:492-503.

12.Jameson, D.M. and W.H. Sawyer. 1995. Fluorescence anisotropy applied to biomolecular interactions. Methods Enzymol. 246:283-300.

13.Jolley, M.E. 1981. Fluorescence polarization immunoassay for the determination of therapeutic drug levels in human plasma. J. Anal. Toxicol. 5:236-240.

14.Lan, N., R.P. Howrey, S.-W. Lee, C.A. Smitha and B.A. Sullenger. 1998. Ribozyme-mediated repair of sickle beta-globin mRNAs in erythrocyte precursors. Science 280:1593-1596.

15.Lewin, A.S., K.A. Drenser, W.W. Hauswirth, S. Nishikawa, D. Yasumura, J.G. Flannery and M.M. LaVail. 1998. Ribozyme rescue of photoreceptor cells in a transgenic rat model of autosomal dominant retinitis pigmentosa. Nat. Med. 4:967-971.

16.Lundbald, J.R., M. Laurance and R.H. Goodman. 1996. Fluorescence polarization analysis of protein-DNA and protein-protein interactions. Mol. Endocrinol. 10:607-612.

17.Murakami, A., M. Nakaura, Y. Nakatsuji, S. Nagahara, Q. Tran-Cong and K. Makino. 1991. Fluorescent-labeled oligonucleotide probes: detection of hybrid formation in solution by fluorescence polarization spectroscopy. Nucleic Acids Res. 19:4097-5102.

18.Nasir, M.S. and M.E. Jolley. 1999. Fluorescence polarization: an analytical tool for immunoassay and drug discovery. Comb. Chem. and High-Throughput Screen 2:177-190.

19.Perrin, F. 1926. Polarization de la lumiere de fluorescence. Vie moyenne de molecules dans l'etat excite. J. Phys. Radium 7:390-401.

20.Singh, K.K., R. Parwaresch and G. Krupp. 1999. Rapid kinetic characterization of hammerhead ribozymes by real-time monitoring of fluorescent resonance energy transfer (FRET). RNA 5:1348-1356.

21.Singh, K.K., P. Schluff, L. Lehnert and G. Krupp. 1996. Design of hammerhead ribozymes to distinguish single base changes in substrate RNA. Antisense and Nucleic Acid Drug Dev. 6:165-168.

22.Spears, P., C.P. Linn, D.L. Woodard and G.T. Walker. 1997. Simultaneous strand displacement amplification and fluorescence polarization detection of Chlamydia trachomatis DNA. Anal. Biochem. 247:130-137.

23.Walker, T.W., C.P. Linn and J.G. Nadeau. 1996. DNA detection by strand displacement amplification and fluorescence polarization with signal enhancement using a DNA binding protein. Nucleic Acids Res. 24:348-353.

24.Walker, G.T., J.G. Nadeau, C.P. Linn, R.F. Devlin and W. Dandliker. 1996. Strand displacement amplification (SDA) and transientstate fluorescence polarization detection of Mycobacterium tuberculosis DNA. Clin. Chem. 42:9-13.

25.Weber, G. 1953. Rotational Brownian motion and polarization of the fluorescence of solutions. Adv. Protein Chem. 8:415.

26.Yonemura, K. and H. Maeda. 1982. A new assay method for DNase by fluorescence polarization and fluorescence intensity using DNA-ethidium bromide complex as a sensitive substrate. J. Biochem. (Tokyo) 92:12971303.

Received 1 November 1999; accepted 25 February 2000.

\author{
Address correspondence to: \\ Dr. Kumud K. Singh \\ Department of Molecular Biology and \\ The Skaggs Institute for Chemical Biology \\ The Scripps Research Institute \\ MB 35, 10550 North Torrey Pines Road \\ La Jolla, CA 92037, USA \\ e-mail:kumudks@scripps.edu
}

